\title{
La fusion-réorganisation dans un groupe financier. Une analyse des facteurs de risques psychosociaux
}

The impact of a financial company restructuring on working conditions and the impacts with professional stress

Djamel Messaoudi

\section{CpenEdition}

\section{Journals}

Édition électronique

URL : http://journals.openedition.org/travailemploi/5166

DOI : 10.4000/travailemploi.5166

ISSN : 1775-416X

Éditeur

DARES - Ministère du Travail

Édition imprimée

Date de publication : 15 juin 2011

Pagination : 17-33

ISSN : 0224-4365

Référence électronique

Djamel Messaoudi, «La fusion-réorganisation dans un groupe financier. Une analyse des facteurs de risques psychosociaux », Travail et Emploi [En ligne], 126 | avril-juin 2011, mis en ligne le 24 juin 2014 consulté le 19 avril 2019. URL : http://journals.openedition.org/travailemploi/5166 ; DOI : 10.4000/ travailemploi.5166 


\title{
La fusion-réorganisation dans un groupe financier. Une analyse des facteurs de risques psychosociaux
}

\author{
Djamel Messaoudi ${ }^{(*)}$
}

\begin{abstract}
Cet article propose une analyse de l'impact d'une restructuration de type fusion-réorganisation sur les situations de travail des salariés. Dans ce contexte, l'auteur tente plus précisément d'établir un lien entre les conditions de travail et le stress professionnel. En s'appuyant sur une enquête auprès des salariés de sept établissements financiers qui ont opéré une fusion-réorganisation entre 2007 et 2008, il identifie les principaux facteurs de risques psychosociaux et leur contribution au stress. L'enquête montre qu'une partie des salariés cumule plusieurs facteurs de risques psychosociaux et que le stress observé chez cette population est le plus élevé. L'examen de l'«origine» du stress met en avant une interdépendance de certains facteurs, comme le manque d'autonomie, la charge de travail et les exigences psychologiques, qui peut expliquer leur cumul pour les catégories socioprofessionnelles les plus touchées par le changement organisationnel. Cette interaction des facteurs de risque est susceptible de limiter le contrôle perçu des salariés et de réduire l'efficacité de leurs stratégies de coping au sens de Lazarus et Folkman (1984). Le niveau de stress serait amplifié par cette interdépendance des facteurs de risques psychosociaux.
\end{abstract}

Pour faire face à l'intensification de la concurrence dans le marché bancaire et des assurances, plusieurs groupes, notamment les réseaux mutualistes (Richez-Battesti, Gianfaldoni, 2005), ont mené des fusions-acquisitions dans le but d'atteindre une taille optimale synonyme d'économies d'échelle et de variété. Ces fusions se sont souvent accompagnées d'une rationalisation de l'organisation de travail susceptible d'améliorer l'efficience productive (ORY, JAEGER, GURTNER, 2006). Ces restructurations visentune organisation du travail souple, pilotée par des processus soumis à une adaptation constante aux diverses contraintes internes et externes. Ces évolutions ne sont pas sans conséquences sur les conditions de travail. Outre la suppression d'emplois souvent génératrice de surcharge de travail (HIRES, 2010; OIT, 2001), la réorganisation modifie les conditions de travail et crée une exigence d'adaptation à de nouvelles règles, à de nouveaux produits ou à de nouveaux marchés.

Les modifications de l'environnement de travail, sources de nouvelles exigences professionnelles, peuvent être assimilées à des facteurs de risques psychosociaux $^{(1)}$ (RPS par la suite). C'est ce que

(*) ORSEU, Office européen de recherche et de conseil en relations sociales; d.messaoudi@orseu.com

L'auteur remercie Nicolas Farvaque et Jean-Luc Bizeur pour leurs remarques.

(1) «Les risques psychosociaux sont des risques professionnels, dont le stress, qui portent atteinte à l'intégrité physique et à la santé mentale des salariés» (Ministère du travail, de la solidarité et de la fonction publique). Les facteurs de RPS recouvrent, selon l'Inserm, «les contraintes psychologiques, sociales et relationnelles dérivées de l'organisation du travail» (INSERM, 2011, p 25). propose d'analyser cet article en s'appuyant sur une enquête réalisée auprès d'un échantillon de 3347 salariés de sept établissements financiers qui ont opéré une fusion réorganisation ( $c f$. encadré 1). Cette enquête met en évidence une interdépendance des facteurs de RPS dans le sens où chaque facteur contribue à l'émergence ou à l'intensité du risque d'un autre facteur. Nous nous interrogeons sur l'impact d'une telle interdépendance sur les aptitudes des salariés à faire face au stress professionnel.

Pour comprendre cet impact, nous adoptons l'approche transactionnelle de Richard S. LAZARUS et Susan FolKMAN (1984) selon laquelle le stress est le résultat d'un déséquilibre entre les contraintes de l'environnement et le contrôle perçu par l'individu. Selon cette approche, l'individu met en œuvre une régulation séquentielle de ses contraintes en adoptant des stratégies d'ajustement ou de coping. À chaque séquence, un salarié en situation de stress perçu évalue ses contraintes et ajuste ses réponses en cherchant les ressources dans son environnement de travail (Ponnelle, LANCRY, 2007). La situation reste stressante durant tout ce processus d'ajustement jusqu'à l'aboutissement d'une solution d'équilibre lui permettant de contrôler ou de modifier ses contraintes.

L'échec de ce processus d'ajustement, ou sa durée qui est synonyme d'un stress permanent, serait amplifié par l'interdépendance des facteurs de RPS. En effet, le fait d'être confronté à une multitude de facteurs de risque en même temps limite l'efficacité des stratégies d'ajustement, ce qui maintient la situation de stress perçu. C'est ce que nous essayons 


\section{Encadré 1 \\ Description de l'enquête et présentation de l'entreprise}

Cet article s'appuie sur une enquête sur les RPS menée par le cabinet Orseu (1) à la demande du Comité d'hygiène, de sécurité et des conditions de travail (CHSCT) de trois entités régionales issues de la fusion de sept filiales d'un groupe financier mutualiste : l'entité de la région Centre (fusion de deux anciennes filiales régionales), l'entité de la région Ouest (trois anciennes filiales) et l'entité de la région Sud (deux anciennes filiales).

\section{Le groupe}

Le groupe est structuré en trois niveaux : le niveau local, généralement le département, constitué de filiales de statut coopératif sans activité financière. Chaque filiale locale est composée d'un réseau d'agences et d'un site de fonctions de support. Le niveau régional est représenté par des sociétés coopératives à activité financière dont le capital est détenu par les sociétés locales. Le troisième niveau est l'organe central de statut de SA dont le capital est majoritairement détenu par les entités régionales. L'organe central définit la stratégie économique et commerciale déclinée au niveau local par l'intermédiaire des entités régionales. Chaque entité adapte les lignes stratégiques aux conditions du marché local et définit son plan d'actions commercial et le calendrier de sa réalisation. Si les entités régionales ont une autonomie dans leurs politiques commerciales, les objectifs économiques sont en revanche imposés par l'organe central. Avant les fusions, chaque filiale disposait d'une certaine autonomie dans la déclinaison du plan d'actions régional, de ses propres ressources organisationnelles et de ses règles de fonctionnement configurées par une histoire locale des relations de travail. La fusion impose la mutualisation de ressources organisationnelles et l'harmonisation des règles et des plans d'actions.

\section{L'enquête}

Les CHSCT des trois entités régionales ont souhaité une expertise approfondie sur les conséquences de la fusion sur les conditions de travail. Plus précisément, il s'agit d'identifier les facteurs de RPS et de mesurer leur impact sur les salariés. La finalité de l'enquête est de fournir aux partenaires sociaux un diagnostic leur permettant de mettre en place des plans d'actions contre le stress et de prévention des RPS. L'enquête est effectuée à travers un questionnaire de 115 questions et 143 entretiens avec les salariés, les managers ainsi qu'avec les représentants du personnel et les médecins du travail. Ces entretiens sont effectués in situ par une équipe composée de 2 psychologues de travail, 2 économistes, 2 spécialistes en $\mathrm{RH}$ et une juriste.

L'enquête s'est déroulée en 2009 , soit un à deux ans après les fusions. Les répondants sont uniquement ceux dont l'emploi est maintenu. L'objectif de l'enquête est de relever l'impact de la restructuration sur les conditions de travail et les relations au travail. Dans le cadre de cet article, nous avons extrait les résultats de 54 questions en lien avec notre problématique. Ces questions sont réparties en trois sections : la mesure du stress perçu (10 questions); l'identification des facteurs de RPS (33 questions), les variables socioprofessionnelles (11 questions).

Sur un effectif total de 6167 salariés des trois établissements, 3347 ont rempli le questionnaire, soit un taux de réponse de $54,2 \%$. Les taux de réponse selon les fonctions, le sexe, l'âge et l'ancienneté sont proches (51\% à $55 \%$ ). Par ailleurs, le taux de réponse chez les cadres est relativement plus important, soit $60 \%$ contre $54 \%$ chez les agents de maîtrise et $46 \%$ chez les employés. Les fonctions support représentent $28,3 \%$ et les fonctions commerciales $71,7 \%$. Nous comptons $50,4 \%$ de femmes et $49,6 \%$ d'hommes. $28,6 \%$ des répondants sont âgés de moins de 35 ans, $32 \%$ ont entre 35 et 45 ans et $39,4 \%$ plus de 45 ans. Les employés représentent $25,8 \%$, les agents de maîtrise $34 \%$, les cadres administratifs et conseillers auprès des professionnels $18 \%$ et les cadres managers $23 \%$.

(1) Cabinet agréé par le ministère du Travail pour les expertises CHSCT.

de montrer dans cet article en nous intéressant aux stratégies de coping des salariés confrontés à cette interdépendance des facteurs de RPS.

\section{La fusion interne et son impact sur l'organisation, l'emploi et le travail}

\section{Des réorganisations successives synonymes d'instabilité de l'organisation du travail}

La fusion-réorganisation étudiée ici peut être assimilée à la rationalisation du facteur organisationnel dans le but d'éliminer les sources d'inefficience productive (LEINBENSTEIN, 1972). Cette rationalisation n'est pas spécifique aux trois entités étudiées ici. Elle est une tendance générale dans le milieu bancaire et des assurances depuis deux décennies. En 10 ans, le nombre d'établissements de crédit en France a diminué de $37 \%$, dont plus de la moitié a disparu dans le cadre de fusions ou de restructurations internes, d'après le Comité des établissements de crédit et des entreprises d'investissement (CECEI, 2009). Ainsi, le réseau du Crédit Agricole est passé de 54 banques à 40 entre 1999 et 2008, le réseau du Crédit Mutuel de 24 à 18, celui des Banques Populaires de 42 à 28, et celui des Caisses d'épargne de 34 à 17. Ces restructurations sont des réponses aux impératifs de la concurrence et de la demande. Étant donné la spécificité de la fonction des banques et assurances selon laquelle la productivité est concentrée dans

-18 - Travail et Emploi $n^{\circ} 126$ 
le facteur organisationnel (PASTRÉ, 1993; THENET, Guillouzo, 2003), la recherche de l'efficience implique donc son optimisation permanente, ce qui explique la récurrence des restructurations dans le milieu bancaire (ORY, JAEGER, GURTNER, 2006).

$\mathrm{La}$ fusion-réorganisation des sept filiales examinées dans l'article illustre ce processus de changements appelant d'autres changements. En effet, la nouvelle organisation est pensée dans le cadre du management par les processus. Ce management consiste à modéliser l'organisation du travail sous formes de process plus ou moins séparés dans le but de mieux maitriser leur fonctionnement et d'améliorer leur qualité. Elle se traduit par une évolution permanente des exigences internes et externes.

Certaines activités comme les services informatiques, les services d'assistance, les opérations de saisie ou la gestion des relations avec les clients ont fait l'objet de regroupements au sein des départements dédiés. Il est plus facile alors d'opérer des changements ciblés sur ces entités sans toucher à l'ensemble de l'organisation. Le fonctionnement de certaines activités a été transformé (opérations de saisie) tandis que d'autres activités ont été mutualisées au sein des plateformes nationales ou locales (l'assistance au réseau, les systèmes informatiques et les appels téléphoniques).

Dans le réseau, la logique est différente. Après la fermeture de quelques agences non rentables et l'organisation des mobilités régionales, l'architecture des agences a été modifiée dans le but d'intégrer davantage de fonctionnalités et d'automates rendant le client plus autonome. En 2009, ce modèle d'agence a subi une deuxième réorganisation où l'utilisation des canaux téléphoniques et le réseau internet sont privilégiés par rapport à la relation directe avec le client. Désormais, certaines agences sont ouvertes la matinée au grand public tandis que l'après-midi est réservée aux rendez-vous sélectionnés. À la date de l'enquête, une révision des amplitudes horaires était en cours de négociation entre les partenaires sociaux.

\section{Rationalisation de l'organisation par la mutualisation du travail et la suppression d'emplois}

$\mathrm{La}$ première rationalisation organisationnelle immédiate dans le cadre de la fusion est la mutualisation des fonctions de support entre les anciennes filiales locales dans le but d'alléger leur effectif salarial. L'objectif est de consolider l'équilibre organisationnel autour du ratio de 3 salariés des fonctions support pour 7 commerciaux à court terme et de tendre progressivement vers un ratio de 1 salarié support pour 3 commerciaux. La mutualisation de ces fonctions a conduit à la fermeture de quatre établissements sièges sur les sept préexistants, avec le maintien de quelques pôles d'activité dans certains des établissements supprimés. Parallèlement, les services informatiques ont opéré leur migration vers un site national. Les réseaux d'agences ont été redéployés de façon à optimiser la couverture du marché local, impliquant des mobilités intra-réseaux.

$\mathrm{Au}$ total, nous avons estimé l'impact de cette fusion à 490 postes supprimés dans les activités du support, ce qui représente entre 7 et $10 \%$ de l'effectif selon les entités. Ces suppressions de postes sont en partie des départs en préretraite. L'autre partie des salariés concernés s'est vue proposer une mobilité géographique ou professionnelle vers les métiers de vente et de services aux clients.

\section{Une réorganisation interne des activités qui modifie les situations de travail}

La création des nouvelles entités s'est accompagnée de la redéfinition et de l'harmonisation des métiers et des fonctions qui ont donné lieu à une nouvelle répartition des activités entre les équipes et entre les salariés. Au sein du réseau, la spécialisation par type de clientèle est privilégiée au détriment de la spécialisation par type de produits. Cela implique pour certains une adaptation à de nouveaux produits financiers encadrés par des règles spécifiques. Leur maîtrise nécessite un effort d'apprentissage individuel et collectif puisque les processus de validation de la vente mobilisent souvent plusieurs acteurs. De plus, les amplitudes de travail devaient être modifiées du fait du développement des services en ligne nécessitant une interactivité croissante avec les clients.

Le fonctionnement interne a fait l'objet d'une «professionnalisation» par l'unification et l'introduction de nouvelles règles. La standardisation des procédures internes devait permettre aux filiales fusionnées d'améliorer leur efficacité organisationnelle et aux salariés de gagner en productivité. Sans préjuger de l'efficacité de ces nouveaux modes opératoires unifiés, ces derniers ont bouleversé les habitudes de travail des salariés. Selon eux, cette évolution consacre la domination du travail prescrit et limite leur autonomie. Leurs interactions «informelles», sources d'une meilleure fluidité des processus de prise de décision, se trouvent encadrées, voire interdites par ces règles. Ceci est le cas par exemple des relations interpersonnelles entre les salariés du siège et ceux du support. Désormais, les commerciaux ne doivent plus appeler leurs «amis» du siège en cas de besoin sur la réglementation par exemple; ils doivent solliciter le Centre de ressources régional nouvellement créé pour ce besoin. Selon les salariés, ce nouveau process est moins efficace que les relations interpersonnelles dans la mesure où la multiplicité d'interlocuteurs au sein du Centre perturbe la continuité de la relation de service d'assistance. 
Des niveaux de stress disparates selon les métiers et les catégories socioprofessionnelles

Pour mesurer le stress, nous utilisons une échelle dite PSS ou Perceived Stress Scale ( $c f$. encadré 2). Cette échelle de mesure est un indicateur de débordement émotionnel de l'individu (COHEN et al., 1983). Plus la valeur de cet indicateur est élevée, plus le salarié est sujet au stress. La valeur moyenne de la PSS de l'échantillon est de 19,24 sur une échelle de 0 à 40 . Précisons que les valeurs individuelles de la PSS n'ont de sens que par leur comparaison à la moyenne de l'échantillon.

On constate des disparités importantes des niveaux de stress. Ce dernier varie d'un individu à un autre de $38 \%$ en moyenne (écart type relatif à la moyenne). Cette disparité d'exposition au stress peut être expliquée par les aptitudes personnelles des salariés à gérer les situations de tension (Neufeld, Paterson, 1989), mais ces capacités sont aussi dépendantes de la qualité de l'organisation et des relations de travail. Comme le note Tim J. Newton (1989), les stratégies d'ajustement au stress peuvent être apprises et conditionnées par des environnements particuliers. L'entreprise comme structure de valeurs et de normes est un environnement dans lequel ces stratégies se construisent (Bellotto, Colautti, 1987). Les facteurs de RPS analysés dans le point suivant permettent de qualifier cet environnement.
Les résultats de l'enquête mettent en effet en évidence une distinction entre les fonctions de support (activités du siège) et les fonctions commerciales (activités des agences). Plus d'un tiers des commerciaux présentent un stress élevé ou très élevé contre un salarié sur cinq des fonctions de support. Ces deux populations ont chacune leurs spécificités en termes d'organisation de travail, de règles de fonctionnement ou de rémunération. Les changements opérés dans le cadre de la fusion-réorganisation et leurs conséquences sont de natures différentes entre les deux populations, que nous avons par conséquent choisi d'analyser séparément.

La validation scientifique de la PSS a montré une corrélation négative entre le stress perçu et le niveau de qualification (QuINTARD, 1994). Nous obtenons la même corrélation concernant notre enquête puisque le niveau de stress décline avec la catégorie socio-professionnelle ( $c f$. tableau 1). Les cadres apparaissent en majorité peu ou pas stressés alors qu'une partie importante des employés, des techniciens et des agents de maîtrise présentent un stress élevé ou très élevé. En revanche, à qualification égale (estimations non reproduites ici), la distribution des niveaux de stress est pratiquement identique entre les salariés du support et ceux du réseau. Ceci s'explique par le fait que la restructuration a majoritairement eu des conséquences sur les basses qualifications, quelle que soit la fonction exercée.

Graphique 1 : La distribution des scores de la PSS (Moyenne : 19,24; Écart-type : 7,37)

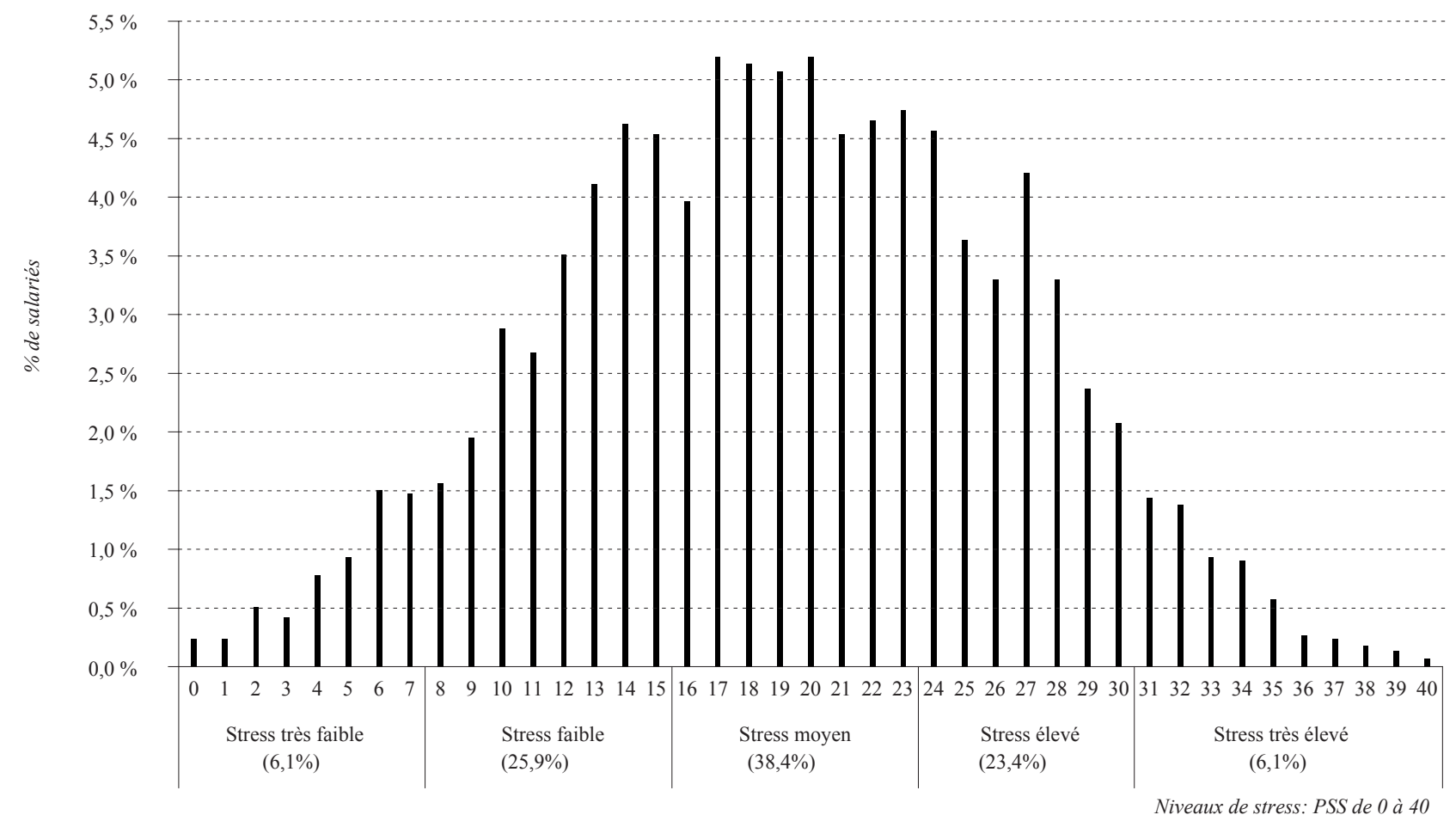

Lecture : $6,1 \%$ des répondants à l'enquête présentent un stress très faible, soit un score de la PSS $\leq 7$.

Champ : Salariés d'un groupe financier ayant opéré une fusion de ses filiales régionales (cf. encadré 1).

Source : Enquête ORSEU ( $c f$. encadré 1).

- 20 - Travail et Emploi $n^{\circ} 126$ 
Dans le support, les qualifications les plus touchées par les stress sont les employés et techniciens (catégorie 1). Plus de $31 \%$ de cette catégorie y est stressée, contre une moyenne de $20 \%$ dans le support. Dans le réseau, ce sont les conseillers commerciaux (agents de maîtrise) qui sont les plus touchés avec $40 \%$ de salariés stressés. D’après nos observations, cette catégorie est de fait la plus affectée par l'évolution du management commercial.

Tableau 1 : Niveaux de stress selon les caractéristiques des salariés et de leur emploi

\begin{tabular}{|ll|c|c|c|}
\hline \multicolumn{1}{|c|}{ Modalités } & Faible ou très faible & Moyen & Élevé et très élevé \\
\hline Domaine & Fonctions support & 44,8 & 35,3 & 19,8 \\
& Fonctions commerciales & 27,0 & 39,6 & $\mathbf{3 3 , 4}$ \\
\hline Sexe & Hommes & 35,3 & 39,2 & 25,5 \\
& Femmes & 28,9 & 37,8 & $\mathbf{3 3 , 3}$ \\
\hline Âge & Moins de 35 ans & 33,6 & 39,4 & 27,0 \\
& 35 à 44 ans & 29,6 & 37,8 & $\mathbf{3 2 , 6}$ \\
& 45 ans et plus & 32,8 & 38,5 & 28,7 \\
\hline Ancienneté & Moins de 5 ans & 39,9 & 35,3 & 24,8 \\
& 5 à moins de 10 ans & 29,4 & 42,3 & $\mathbf{3 2 , 4}$ \\
\hline Qualification & 31,5 & 36,3 & $\mathbf{3 5 , 5}$ \\
& Plus de 10 ans & Cat. 1 (Employé et technicien) & 28,2 & $\mathbf{3 4 , 6}$ \\
& Cat. 2 (Agent de maîtrise) & 25,4 & 36,4 & 22,9 \\
& Cat. 3 (Cadre et assimilé cadre) & 36,4 & 40,1 & 22,2 \\
\hline & Cat. 4 (Cadre sup. et Dir. d'agence) & 40,2 & 37,6 & 11,7 \\
\hline
\end{tabular}

Note : Les nombres en gras correspondent aux situations où le stress élevé ou très élevé est plus fréquent.

Lecture : 44,8 \% des salariés dans les fonctions de support présentent un stress faible ou très faible.

Champ : Salariés d'un groupe financier ayant opéré une fusion de ses filiales régionales ( $c f$. encadré 1).

Source : Enquête ORSEU ( $c f$. encadré 1).

\section{Encadré 2}

\section{L'approche transactionnelle et l'échelle de mesure du stress dite PSS}

Selon LazARUs et FolKman (1984, p. 19), le stress se définit comme "une transaction particulière entre un individu et une situation dans laquelle celle-ci est évaluée comme débordant ses ressources et pouvant mettre en danger son bien-être". Le stress dans ce modèle transactionnel est le produit entre la réalité objective et la perception qu'en a l'individu. Cette perception est déterminée par l'écart entre une évaluation primaire des contraintes et une évaluation secondaire des ressources (LAZARUS, FolKMAN, 1984; SARAFINO, 1994). Dans l'évaluation primaire, l'individu identifie si la situation est stressante et en mesure la gravité, le risque, l'enjeu, etc. II s'en suit une évaluation secondaire consistant à apprécier ses ressources (contrôle perçu et soutien social perçu). Suite à cette double évaluation, le salarié détermine un ensemble de stratégies pour contrôler ou modifier la situation (stratégie de coping). Ces stratégies sont un ensemble d'efforts cognitifs et comportementaux (actions) pour gérer les exigences identifiées comme excédant ses ressources jusqu'à ce que le salarié arrive à l'équilibre entre ressources et contraintes. La situation de stress perdure durant ce processus de déploiement du coping. L'entreprise peut aider ses salariés à réguler les exigences de leur travail notamment en mettant à leur disposition des ressources permettant d'activer un coping centré sur le problème et non pas sur les émotions.

\section{La mesure du stress par l'échelle dite PSS}

Pour mesurer le stress, nous utilisons l'échelle dite de Stress Perçu ou PSS (Perceived Stress Scale) construite par Cohen et al., (1983). Cette échelle permet de rendre opérationnelle la conception transactionnelle du stress (BelLINGHAusen et al., 2009). Conformément à cette approche, la PSS mesure le sentiment de débordement issu de l'évaluation primaire et secondaire.

La PSS utilisée dans cet article est la version française à 10 items qui présente des qualités psychométriques satisfaisantes selon Lisa Bellinghausen et al. (op. cit.). Selon de nombreuses études (CoHEN, WiLLIAMSON, 1988; KoLECK et al., 2002), les items constitutifs de la PSS présentent une bonne cohérence interne : le coefficient alpha de Cronbach de 0,78 et 0,87 . Plus ce coefficient tend vers 1 plus l'homogénéité des réponses est parfaite. La PSS calculée pour notre échantillon présente un coefficient de cohérence de 0,9 . 
La plupart des études ont mis en évidence l'existence de deux facteurs dans l'échelle de la PSS (BELLINGHAUSEN et al., idem). Concernant la version française de cette échelle, les deux facteurs sont interprétés successivement en termes de vulnérabilité perçue et de contrôle perçu (GADEA, CERCLE, 2004). La validation factorielle de la PSS menée par Bellinghausen et al. (idem) traduit les deux facteurs en termes de perception de débordement et d'efficacité personnelle perçue.

La PSS propose aux individus d'évaluer sur une période d'un mois, la fréquence des situations perçues comme stressantes suivant qu'elles soient évaluées comme incontrôlables, imprévisibles et menaçantes. Les items constitutifs de la PSS correspondent à 10 situations proposées à l'auto-évaluation (cf. annexe). Exemple : "au cours du dernier mois, à quelle fréquence vous êtes-vous senti dépassés par tout ce que vous aviez à faire? ". Les propositions de réponse sont : assez souvent, souvent, parfois, presque jamais, jamais. Chaque réponse correspond à un score de 0 (jamais) à 4 (assez souvent). L'addition des scores pour les dix questions nous donne le score de la PSS de 0 et 40. Plus ce score est élevé, plus le stress perçu par l'individu est élevé.

La PSS mesure le stress de manière générale et non de manière spécifique. Elle n'est pas conçue pour repérer l'origine du stress et ne décrit pas les situations de détresse psychologique. Elle décrit plutôt le contrôle perçu sur les événements (CoHen et al., 1983). Le croisement de la PSS avec les facteurs de RPS nous permet de caractériser les origines de stress des salariés interrogés.

\section{La classification des scores de la PSS}

La PSS ne définit pas le seuil à partir duquel on peut dire qu'un salarié est stressé ou non. Pour comparer les niveaux de stress, nous répartissons les valeurs de la PSS en 5 classes, chacune correspondant à un niveau de stress. Pour construire cette classification, nous utilisons les paramètres de la loi normale centrée et réduite.

- stress très faible : PSS $<\mu-1,5 \sigma$ ( $\mu$ : PSS moyenne et $\sigma$ : écart type);

- stress faible : $\mu-1,5 \sigma<$ PSS $<\mu-0,5 \sigma$;

- stress moyen : $\mu-0,5 \sigma<$ PSS $<\mu+0,5 \sigma$

- stress élevé : $\mu+0,5 \sigma<$ PSS $<\mu+1,5 \sigma$;

- stress très élevé : PSS $>\mu+1,5$.

Ces niveaux n'ont de sens que comparativement à la moyenne de l'échantillon. Ainsi, un niveau "élevé» correspond à une PSS supérieur au niveau défini comme «moyen ».

Tableau 2 : Les principaux facteurs de Risques Psychosociaux identifiés dans l'enquête

\begin{tabular}{|l|l|l|}
\hline \multicolumn{1}{|c|}{ Dimensions } & \multicolumn{1}{c|}{ Facteurs de RPS } & \multicolumn{1}{c|}{ Items (questions) } \\
\hline \multirow{2}{*}{$\begin{array}{l}\text { Exigences de } \\
\text { travail }\end{array}$} & Charge de travail élevée & Quantité, qualité, rapidité, complexité du travail \\
\cline { 2 - 3 } & $\begin{array}{l}\text { Exigences psychologiques du } \\
\text { travail élevées }\end{array}$ & $\begin{array}{l}\text { Adaptation, réalisabilité des objectifs, opposition objectif/devoir de conseil, } \\
\text { responsabilité }\end{array}$ \\
\hline $\begin{array}{l}\text { Ressources } \\
\text { organisationnelles }\end{array}$ & $\begin{array}{l}\text { Répartition du travail } \\
\text { contraignante }\end{array}$ & $\begin{array}{l}\text { Remplacement des absences, efficacité organisationnelle, clarté des } \\
\text { attributions }\end{array}$ \\
\cline { 2 - 3 } & $\begin{array}{l}\text { Processus et procédures de travail } \\
\text { contraignantes }\end{array}$ & $\begin{array}{l}\text { Circulation de l'information, qualité des procédures, coordination entre les } \\
\text { services }\end{array}$ \\
\hline $\begin{array}{l}\text { Ressources } \\
\text { individuelles }\end{array}$ & Faible autonomie & $\begin{array}{l}\text { Possibilité de planifier et de prioriser les tâches, d'interrompre le travail; } \\
\text { autonomie de décision, liberté dans le travail, confiance de la hiérarchie }\end{array}$ \\
\cline { 2 - 3 } & Faible soutien social & Tensions au travail, soutien des collègues \\
\hline Incertitude professionnelle & Menace sur l'emploi, perspectives de carrière \\
\hline
\end{tabular}

Note : Les questions et fréquences associées aux différentes modalités de réponse dans l'ensemble de l'échantillon sont présentées plus loin.

Lecture : Les exigences de travail recouvrent la charge de travail et les exigences psychologiques.

Les femmes apparaissent plus stressées que les hommes. Un tiers d'entre elles perçoivent un stress élevé ou très élevé contre un quart des hommes. Cette différence s'explique par le fait que les femmes sont surreprésentées dans les emplois les plus exposés au stress (catégorie 1 et 2) comparativement aux hommes. En effet à qualification et à poste de travail égal, le niveau de stress entre les hommes et les femmes est quasi-identique : $40 \%$ des femmes conseillères commerciales sont stressées versus $39 \%$ des hommes de même fonction ${ }^{(2)}$.

(2) Le score de la PSS chez les conseillers commerciaux femmes est de 21,6 et de 21,4 chez les conseillers hommes.
L'exposition au stress concerne davantage les salariés âgés entre 35 et 45 ans et dont l'ancienneté est supérieure à 10 ans. Plus de $35 \%$ des salariés ayant ces deux caractéristiques sont stressés. En incluant un critère de qualification, on recense même $42 \%$ des salariés de catégorie 1 et 2 , âgés entre 35 et 45 ans, et ayant une ancienneté de plus de 10 ans qui présentent un stress perçu élevé ou très élevé.

Les différences de niveau de stress semblent ainsi être davantage liées à la fonction exercée qu'aux caractéristiques personnelles (âge, ancienneté ou sexe). Nous verrons par la suite que les salariés de catégorie 1 et 2 sont ceux qui sont confrontés à une multiplicité de facteurs de RPS. 


\section{Les facteurs de risques psychosociaux contribuent différemment au stress perçu}

Chacun des sept facteurs de RPS que nous avons identifiés ( $c f$. tableau 2) renvoie à une catégorie de contraintes au travail engendrée par la fusionréorganisation ou à l'impact de celle-ci sur les marges de manœuvre du salarié.

À travers une analyse «toutes choses égales par ailleurs ${ }^{(3)}$, nous estimons la contribution spécifique de chaque facteur de RPS au stress perçu. Le tableau 3 présente la probabilité d'être stressé suivant l'exposition à un facteur donné par comparaison à un salarié de référence peu exposé à ce facteur.

Ce modèle montre que les sept facteurs de RPS sont des facteurs explicatifs du stress perçu dans le réseau alors que dans le support seulement trois facteurs le sont. En revanche, la contribution au stress des trois facteurs explicatifs dans le support est plus importante que celle observée dans le réseau. Ces deux observations appuient notre hypothèse selon laquelle le niveau de stress est amplifié par la conjugaison de plusieurs facteurs de risque. En effet, d'après notre observation des réorganisations, les contraintes induites par la fusion sont moins fortes dans le réseau mais elles sont multiples, alors qu'elles sont plus intenses dans le support mais moins nombreuses. La différence de niveau de stress perçu entre les deux populations semble tout à fait cohérente avec ces observations : elle pourrait donc s'expliquer par le fait que les commerciaux sont plus nombreux à faire face à une conjugaison de plusieurs facteurs de risque que leurs collègues des fonctions support.
Par ailleurs, on mesure une grande disparité des niveaux de stress dans le support. Certains salariés de ces fonctions sont exposés à plus de facteurs que d'autres. La réorganisation n'a en effet pas eu les mêmes conséquences dans les différents services des fonctions support. En isolant les catégories de salariés qui sont confrontées à plus de quatre facteurs de RPS, nous obtenons un niveau élevé de stress dans le support et légèrement plus élevé que celui perçu dans le réseau : la valeur de la PSS est de 28 dans le support et de 27 dans le réseau (la moyenne de l'échantillon étant de 19,4). Plus généralement, nos calculs montrent que le cumul des facteurs de risque amplifie le niveau de stress. Un salarié qui cumule plus de quatre facteurs de RPS présente un niveau de stress perçu de $43 \%$ de plus qu'un salarié exposé à un seul facteur et de $18 \%$ de plus à celui qui est exposé à moins de trois facteurs ${ }^{(4)}$.

Établie statistiquement, l'amplification du stress selon le nombre de facteurs de RPS peut s'expliquer par l'effet cumulatif de leurs contraintes. Une surcharge de travail due aux objectifs prescrits par exemple est une contrainte qui s'ajoute à celle induite par une perte d'autonomie suite à l'évolution du management. Cet effet cumulatif des contraintes renvoie à l'interdépendance des facteurs de RPS.

Le niveau de stress est amplifié par le cumul de facteurs de risque mais aussi par le type des facteurs cumulés. Le modèle logit du tableau 3 montre que certains facteurs augmentent plus fortement le risque de stress que d'autres. Dans le réseau, la charge de travail et la faible autonomie sont les deux facteurs dont la contribution au stress est la plus élevée. Le premier multiplie le risque de stress par 4 et le second par 2,9 (les autres facteurs de risque étant donnés). Selon nos calculs (non détaillés ici), un commercial exposé à ces deux facteurs présente un

Tableau 3 : La probabilité d'être stressé selon l'exposition élevée aux facteurs de RPS

\begin{tabular}{|c|c|c|c|c|}
\hline \multirow[t]{2}{*}{ Facteurs de risque psychosociaux } & \multicolumn{2}{|c|}{ Réseau de commerciaux } & \multicolumn{2}{|c|}{ Fonctions Support (siège) } \\
\hline & Odd-ratio & Significativité & Odd-ratio & Significativité \\
\hline Charge de travail élevée & 4,08 & $* * *$ & 6,57 & $* * *$ \\
\hline Répartition du travail contraignante & 1,39 & $* *$ & 3,37 & $* * *$ \\
\hline Exigences psychologiques élevées & 1,49 & $* * *$ & 1,43 & $n s$ \\
\hline Processus et procédures contraignants & 1,72 & $* * *$ & 1,90 & $n s$ \\
\hline Faible autonomie & 2,90 & $* * *$ & 1,59 & $n s$ \\
\hline Faible soutien social & 1,83 & $* * *$ & 1,68 & $n s$ \\
\hline Incertitude professionnelle & 1,68 & $* * *$ & 3,28 & $* * *$ \\
\hline
\end{tabular}

Note : Ce tableau reprend les résultats de deux régressions testées séparément pour le réseau et pour le support. Les sept facteurs de RPS sont intégrés ensemble dans les deux régressions. Les odds ratio donnent ainsi une approximation du risque relatif de chaque facteur sur la probabilité d'être stressé, sans autre variable de contrôle. S'il est significatif et supérieur à 1 , le facteur $i$ contribue positivement au stress. Seuils de significativité : * : $5 \% ; * *: 1 \% ; * * *: 0,1 \%$.

Lecture : Le risque de stress est multiplié par 4 , à autres facteurs de risque contrôlés, chez un commercial exposé à une charge de travail élevée par rapport à un autre commercial ayant une faible charge de travail.

Champ : Salariés d'un groupe financier ayant opéré une fusion de ses filiales régionales ( $c f$. encadré 1).

Source : Enquête ORSEU ( $c f$. encadré 1).

(3) Au regard de la spécificité des métiers dans chacune des deux fonctions (réseau et support), nous avons choisi de mener une régression logistique séparée. Le tableau 3 présente les résultats des deux régressions.
(4) La PSS pour plus de 4 facteurs cumulés de RPS est de 27,2 contre 23,07 pour 2 à 3 facteurs et 19 pour un seul facteur. 
stress perçu supérieur de $25 \%$ à un salarié exposé à deux facteurs autres que l'autonomie et la charge de travail. Dans le support, le cumul des deux principaux facteurs de stress qui sont la charge de travail et la répartition de l'activité génère un niveau de stress supérieur de $35 \%$ à un salarié qui cumule deux autres types de facteurs. La conjugaison de plusieurs facteurs prépondérants exerce ainsi un effet cumulatif spécifique sur la contrainte perçue nécessitant des ressources importantes et spécifiques pour limiter le risque de stress.

Afin d'éclairer les associations statistiques ainsi établies, nous détaillons dans les sections qui suivent l'exposition des salariés aux différents facteurs de RPS et les mettons en relation avec des observations conduites dans les filiales.

\section{La charge de travail comme résultat de la convergence des facteurs des RPS}

Comme le montre le graphique 2, la perception des salariés de leur charge de travail est majoritairement négative. Cette évaluation concerne l'ensemble des dimensions de ce facteur. Mais les plus sévèrement critiquées sont la complexité du travail et l'exigence en termes de vitesse d'exécution.

Si les différences de charge de travail par âge, ancienneté et sexe sont minimes, celles relatives aux qualifications et au domaine d'activité (entre support et réseau) sont importantes. Les qualifications inférieures sont plus massivement exposées au risque lié à la charge de travail que les cadres ou les managers. Il en est de même pour les commerciaux par rapport aux salariés du support.

Nous l'avons vu (cf. tableau 3), la charge de travail est le facteur le plus important en termes de contribution au risque de stress. Ce risque est multiplié par 4 dans le réseau et par 6,5 dans le support, les autres facteurs de risque étant égaux par ailleurs. Les causes de la surcharge au travail sont multiples : la complexification du travail et des procédures, la pression sur la productivité commerciale, la diminution de l'effectif ou l'absentéisme, la perte d'autonomie, etc. On remarque en outre une forte corrélation entre la charge de travail d'une part, et les facteurs liés aux exigences psychologiques du travail, à la répartition interne de l'activité, aux process et procédures et à l'autonomie d'autre part. Ceci signifie que ces quatre derniers facteurs s'additionnent souvent à la charge de travail perçue. Le facteur lié à la répartition interne de l'activité renvoie à l'intensification du travail induite par la suppression des postes et le non-remplacement des absences notamment dans le support : deux tiers des salariés du support qui affirment que les absences ne sont jamais remplacées estiment que leur charge de travail est élevée. L'influence du facteur «exigences psychologiques» en plus de la charge de travail peut s'exercer notamment à travers la dimension liée aux difficultés à réaliser les objectifs qui est due l'ampleur de ces objectifs et à la complexification des produits. Le sentiment d'incapacité à réaliser les objectifs et celui de débordement de la charge de travail se renforcent mutuellement. Plus de $60 \%$ des salariés qui affirment être en incapacité d'atteindre leurs objectifs estiment que leur charge de travail est élevée.

La perte d'autonomie limite les marges de manœuvre des salariés pour moduler ou pour reporter leur charge de travail. Cette perte d'autonomie est due à l'encadrement des relations de travail par des procédures plus contraignantes auquel s'ajoute la complexification des produits vendus nécessitant des qualifications nouvelles. La perte d'autonomie est l'une des causes de la difficulté des salariés à réaliser leurs objectifs, ce qui est synonyme pour certains d'entre eux de débordement de la charge de travail. Deux tiers des salariés qui subissent une diminution de leur autonomie affirment que leur charge de travail est élevée.

La complexification de process de travail notamment avec l'introduction de projets transversaux

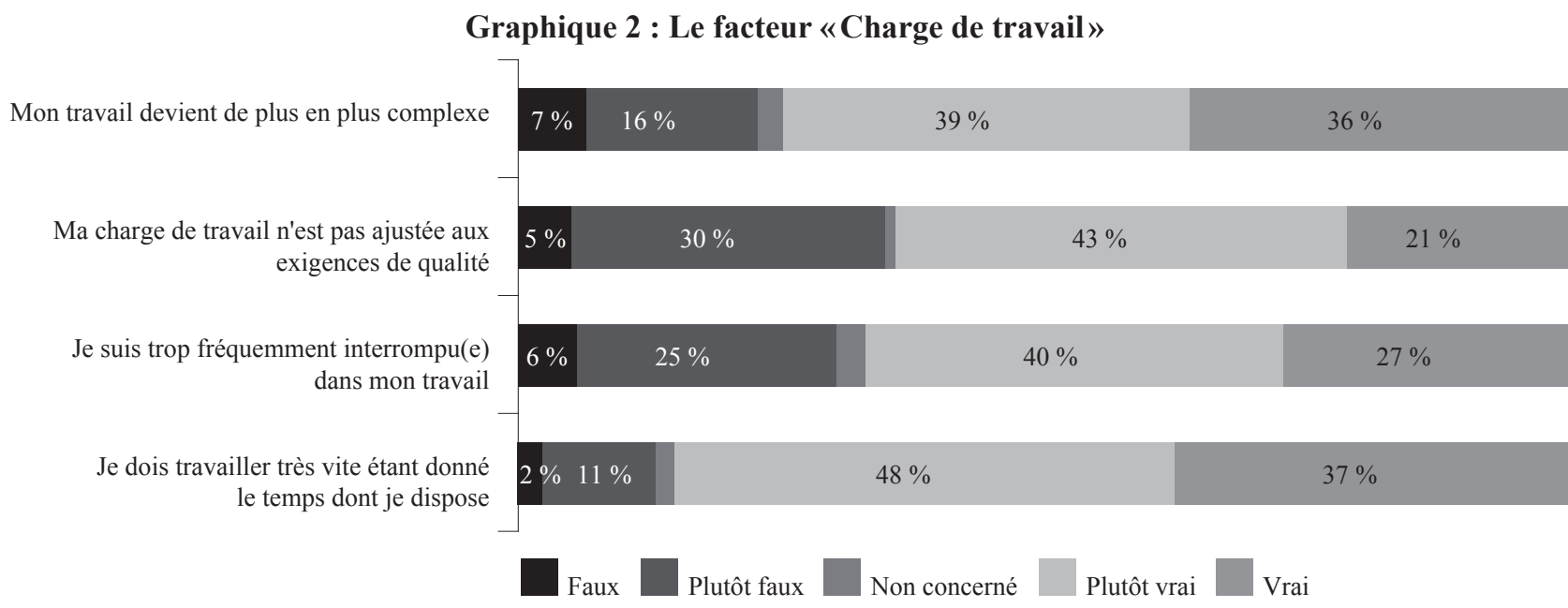

Lecture : Pour $7 \%$ des répondants, l'affirmation «mon travail devient de plus en plus complexe» est fausse.

Champ : Salariés d'un groupe financier ayant opéré une fusion de ses filiales régionales ( $c f$. encadré 1).

Source : Enquête ORSEU ( $c f$. encadré 1). 
exige un effort d'adaptation du salarié à cette nouvelle forme d'organisation de travail. Elle engendre une charge de travail supplémentaire en termes de tâches administratives, de préparation et de participation aux réunions d'équipe, d'autoanalyse des performances, de gestion du portefeuille de clients, etc.

\section{La nouvelle répartition du travail source d'inefficacité organisationnelle selon les salariés}

La nouvelle répartition du travail issue de la réorganisation pose l'épineux problème de l'adaptation des salariés aux nouvelles tâches et celui de la coordination entre les services et les équipes. Historiquement, il y a une difficulté de coordination entre le réseau et les fonctions support que la réorganisation n'a pas résolue. On peut la résumer à un problème de gestion de la prise de décision notamment en ce qui concerne les délais. D'une part, les salariés du siège doivent respecter consciencieusement les procédures et les règles de validation des décisions concernant l'octroi d'un crédit par exemple. D'autre part, les commerciaux subissent la pression du client qu'ils reportent sur les salariés du siège. La fusion-réorganisation ne semble pas régler ce problème puisqu'une majorité des salariés, soit $60 \%$, des commerciaux et $35 \%$ des salariés des fonctions support, affirment que la réorganisation n'a pas amélioré leur coordination. Plus généralement, $68 \%$ des répondants, dont $71 \%$ des commerciaux, affirment que la fusion n'a pas amélioré l'efficacité organisationnelle dans son ensemble.

Précisons aussi que la nouvelle organisation a changé le référentiel métier afin d'harmoniser les emplois. Ce nouveau référentiel n'est pas assez clair selon une partie des salariés, notamment ceux des fonctions support : $31 \%$ de ces derniers affirment que leurs attributions et leurs responsabilités ne sont pas clairement définies. Dans le réseau, cette proportion est de $27 \%$. Le fait que les tâches ne soient pas suffisamment délimitées ne suppose pas que le salarié soit autonome dans la planification de son travail. En effet, $52 \%$ des salariés qui estiment que leurs attributions ne sont pas suffisamment claires disposent d'une faible autonomie perçue, contre une moyenne de l'échantillon de 31,4\%.

Cette critique des changements dans l'organisation et dans les référentiels des métiers renvoie à celle adressée à l'organisation du travail par les process. La confusion des rôles et des responsabilités chez certains salariés et les dysfonctionnements entre le réseau et le support sont associés au mauvais fonctionnement ou à la mauvaise qualité des process mis en place. La nouvelle répartition du travail et la qualité des procédures sont ainsi des facteurs liés.

Sur un autre plan, la nouvelle organisation est jugée incapable de résorber la charge de travail due à l'absentéisme. Plus de $40 \%$ des salariés sont âgés de plus de 45 ans, dont un quart de plus de 50 ans, selon les bilans sociaux des trois entités. L'absentéisme est estimé à 282,3 équivalents temps plein, soit $6,4 \%$ de l'effectif permanent. En plus de la réduction de l'effectif, la nouvelle organisation doit donc gérer ces absences en assurant leur remplacement, faute de quoi la charge de travail des salariés actifs s'accroît. On ignore si l'absentéisme est dû en partie au stress mais selon toute vraisemblance le non remplacement des absences augmente le risque du stress chez les actifs : parmi les 54,5\% des répondants affirmant que l'organisation actuelle ne permet pas d'absorber la charge de travail liée aux absences, $38 \%$ perçoivent un stress élevé ou très élevé contre $29 \%$ en moyenne. Si l'absentéisme peut être une stratégie d'ajustement pour certains salariés, le non-remplacement des absents augmente la charge de travail des présents. Plus de $73 \%$ des salariés dont la charge de travail est élevée affirment que ces absences ne sont pas remplacées.

\section{Graphique 3 : Le facteur «Répartition du travail»}

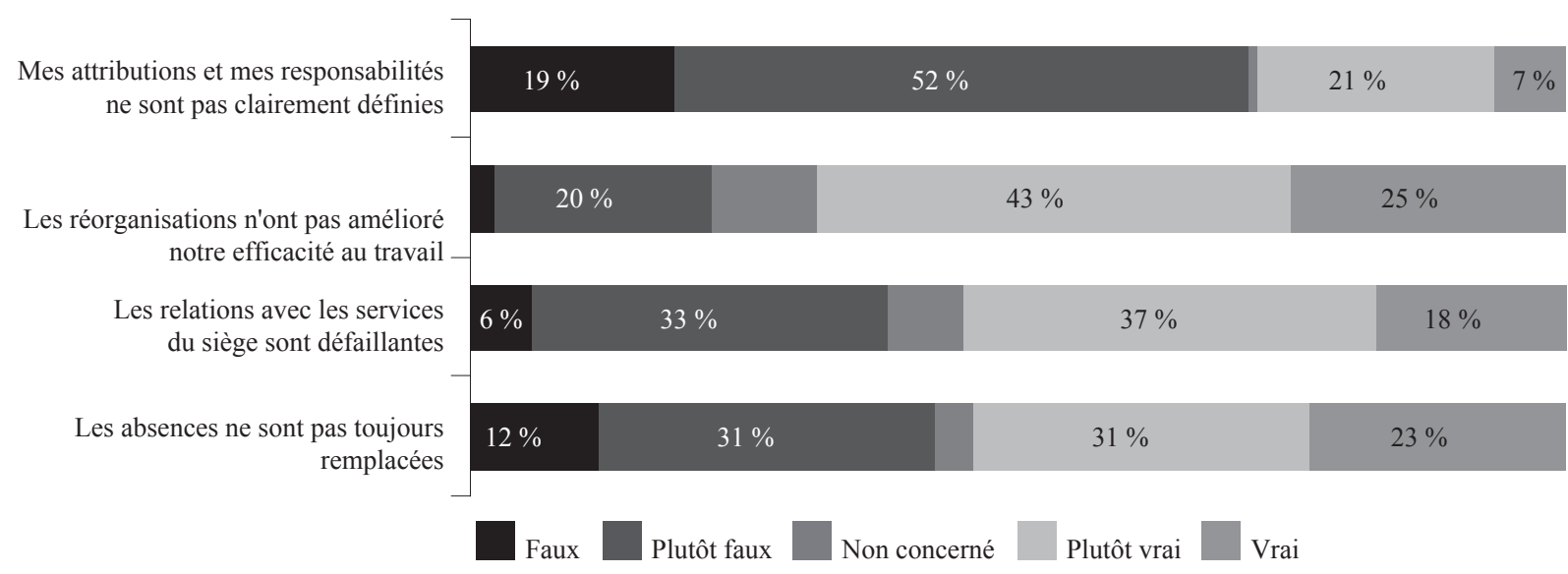

Lecture : Pour $19 \%$ des répondants à l'enquête, l'affirmation «mes attributions et mes responsabilités ne sont pas clairement définies » est fausse. Champ : Salariés d'un groupe financier ayant opéré une fusion de ses filiales régionales ( $c f$. encadré 1).

Source : Enquête ORSEU ( $c f$. encadré 1). 
La nouvelle organisation du travail issue de la fusion est ainsi perçue comme une contrainte par une majorité de salariés plutôt qu'une évolution positive de leurs conditions de travail. Cette contrainte diminue leurs ressources organisationnelles, multipliant ainsi le risque de stress notamment dans le support : le risque de stress y est de ce fait multiplié par 3,3 et par 1,4 dans le réseau.

\section{Une modification des processus internes apportant une nouvelle exigence d'adaptation et limitant l'autonomie dans le travail}

La modification des procédures de travail concerne essentiellement la mise en place d'un reporting de l'activité contraignant, de nouvelles règles de gestion des dossiers-clients ainsi que des nouveaux processus de coordination entre les services. Ces évolutions sont globalement évaluées négativement par les salariés : $60 \%$ affirment que les procédures de travail ne leur permettent pas de bien travailler. Dans le réseau, cette proportion est de $66 \%$. Il y a donc un malaise généralisé à propos des nouvelles procédures mises en place qui est d'autant plus fort quand on sait que la majorité des salariés estiment avoir perdu en autonomie dans leur travail. D'ailleurs, la corrélation entre le facteur autonomie et le facteur processus et procédures est significativement élevée ( $r=$. 48).

Face à ce malaise, quel est le comportement adopté par les salariés? Plus de la moitié des salariés du réseau, dont $60 \%$ des conseillers commerciaux, affirment être amenés à contourner les procédures. Parmi les commerciaux, $72 \%$ éprouvent des difficultés à travailler avec les nouvelles procédures. Pour autant, cette stratégie de contournement ne réduit pas le risque de stress mais au contraire l'augmente. En effet, le niveau de stress (mesuré par la PSS) chez ces salariés est supérieur de $10 \%$ par rapport à la moyenne de l'échantillon. Le risque de stress est multiplié par 1,7 chez les commerciaux subissant la contrainte des nouvelles procédures mises en place.
Le stress associé à ce facteur ressemble à l'issue d'un jeu avec contrainte. En effet, les commerciaux estiment que les procédures sont inefficaces et limitent la possibilité de réalisation de leurs objectifs individuels, ce qui les contraint à contourner ces procédures avec le risque que cela soit considéré comme une faute professionnelle suffisante pour justifier un licenciement. Ne pas réaliser les objectifs assignés est aussi une menace sur leur carrière. Cette menace est renforcée par la pression de la concurrence interne entre les commerciaux, instituée par le benchmarking des performances. Pour certains, contourner les règles afin de réaliser les objectifs est une stratégie dominante, ce qui explique l'importance du nombre de commerciaux à l'avoir adoptée. En revanche, le risque de faute professionnelle associé à cette stratégie est synonyme de stress et d'anxiété.

\section{Une moindre autonomie synonyme de capacité d’ajustement limitée face au stress}

Le rôle de l'autonomie dans l'exercice du travail est souvent ambigu. On peut considérer, qu' a priori, l'autonomie est une ressource qui permet au salarié de moduler sa charge de travail en fonction de ses contraintes. De ce point de vue, elle fait partie des éléments de la stratégie d'ajustement du salarié face au stress (JeX, BeEHR, 1991). Dans d'autres cas, l'autonomie peut être associée non plus à la liberté de moduler le travail prescrit mais davantage à une contrainte organisationnelle. En effet, l'autonomie rime dans certains cas avec isolement et responsabilisation individuelle. Ceci est le cas des fonctions commerciales où le salarié endosse une plus grande responsabilité des fluctuations de sa performance individuelle. Comme le notent Luc Boltanski et Eve Chiapello (1999), l'organisation transfert une plus grande partie du risque vers le salarié en contrepartie d'autonomie accordée ou exigée.

Graphique 4 : Le facteur «Process et procédures de travail»

Les procédures de travail ne me permettent

\footnotetext{
pas de bien travailler
}

Je suis parfois amené(e) à contourner les procédures de travail

Je ne sais pas où trouver l'information ou les interlocuteurs dont j'ai besoin
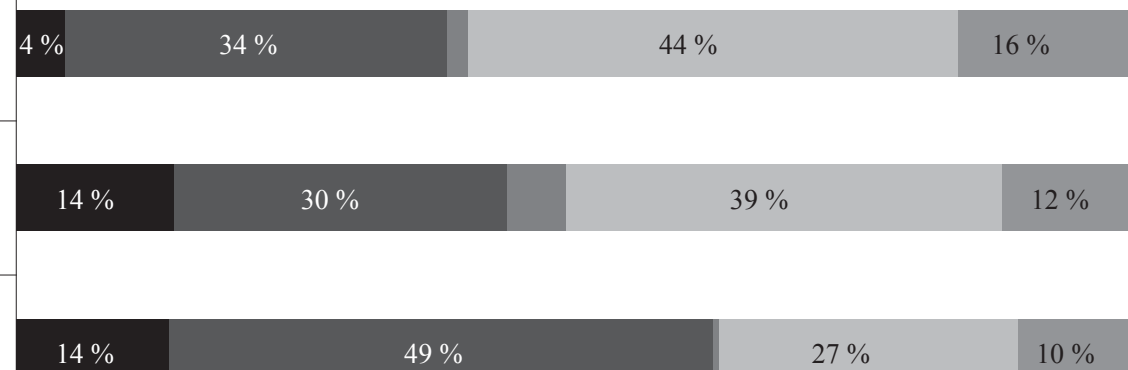

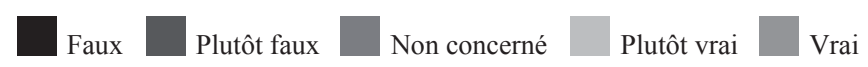

Lecture : Pour $4 \%$ des répondants, l'affirmation «les procédures de travail ne me permettent pas de bien travailler» est fausse.

Champ : Salariés d'un groupe financier ayant opéré une fusion de ses filiales régionales ( $c f$. encadré 1).

Source : Enquête ORSEU ( $c f$. encadré 1).

- 26 - Travail et Emploi $n^{\circ} 126$ 


\section{Graphique 5 : Le facteur «Autonomie au travail»}

Ma hiérarchie ne me fait pas confiance
pour gérer mon travail

Lecture : Pour $28 \%$ des répondants à l'enquête, l'affirmation «ma hiérarchie ne me fait pas confiance pour gérer mon travail» est fausse.

Champ : Salariés d'un groupe financier ayant opéré une fusion de ses filiales régionales ( $c f$. encadré 1).

Source : Enquête ORSEU ( $c f$. encadré 1).

Certaines dimensions de l'autonomie comme la confiance de la hiérarchie ou la possibilité du salarié à hiérarchiser les priorités au travail sont majoritairement jugées positives par les répondants. En revanche, la liberté dans l'exercice du travail, la planification de l'activité ou l'autonomie dans la prise de décision sont jugées négatives par une grande partie de salariés. Presque deux tiers des commerciaux estiment que la planification de leur activité ne leur permet pas de faire face aux imprévus. Cette contrainte est d'autant plus importante quand on sait que l'activité est très fluctuante. En effet, plus de $70 \%$ affirment qu'ils sont «trop fréquemment interrompus dans leur travail», ce qui implique la variabilité dans l'effort de concentration cognitive. En ce qui concerne la prise de décision, $43 \%$ des salariés estiment qu'ils n'ont pas l'autonomie suffisante pour prendre les décisions qu'ils jugent nécessaires pour atteindre leurs objectifs. Plus généralement, une grande partie des répondants, soit $45 \%$, estiment qu'ils manquent de liberté dans l'exercice de leur travail.

Comme nous l'avons constaté dans le tableau 3, la perte d'autonomie est un facteur de stress prépondérant chez les commerciaux. Elle arrive comme deuxième facteur principal de stress après la charge de travail. Le risque de stress est multiplié par 2,9 chez les salariés estimant que leur autonomie a été réduite. En revanche, son impact dans les fonctions support est non significatif. Et ce n'est pas sans conséquence puisque la proportion de salariés affirmant avoir perdu en autonomie dans leur travail est deux fois plus importante dans le réseau que dans le support.

On peut penser que les commerciaux sont sensibles à leur autonomie notamment quand celle-ci met en jeu leur capacité à réaliser les objectifs commerciaux. La complexification des procédures et les nouveaux produits sont également des facteurs qui limitent l'autonomie réelle. On constate d'ailleurs une forte corrélation entre le facteur perte d'autonomie d'une part, les exigences psychologiques, l'organisation du travail et les procédures de travail d'autre part (cf. plus loin graphique 7).

\section{L'exigence psychologique du management par les objectifs et par le benchmarking}

\section{Le poids des objectifs commerciaux}

Pour deux tiers des commerciaux, les objectifs de vente sont en contradiction avec leur « devoir de conseil au client». Cette contradiction est parfois mal vécue notamment pour les conseillers spécialisés dans l'épargne ou dans le conseil en patrimoine. Selon les conseillers que nous avons rencontrés, certains produits risqués ou complexes devaient être présentés au client comme étant rentables et sûrs. Il en est de même pour «l'équipement» $\mathrm{du}$ client en produits annexes comme les divers contrats d'assurance. Ainsi, plutôt que comprendre les besoins du client, le commercial doit davantage susciter ces besoins sans se soucier de l'utilité réelle du produit vendu. Plusieurs d'entre eux subissent psychologiquement ce conflit entre les registres éthique et économique notamment quand le client est une personne fragile (les retraités par exemple). Ce conflit renvoie à ce qu'OrY et al., (2006) qualifient d'opposition entre la «culture financière» et les «valeurs coopératives et mutuelles» qui ont pris corps progressivement avec la mutation des caisses mutuelles dans les années 1990. 


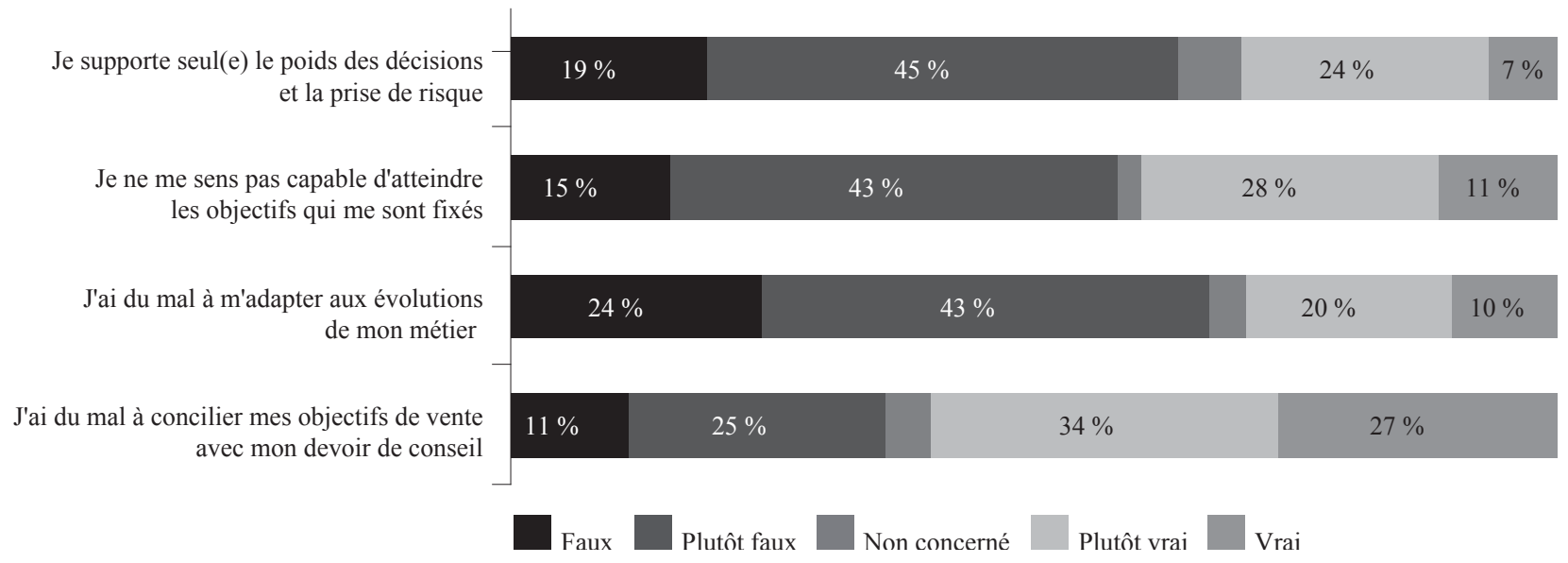

Lecture : Pour 19 \% des répondants à l'enquête, l'affirmation «je supporte seul(e) le poids des décisions et la prise de risque» est fausse. Champ : Salariés d'un groupe financier ayant opéré une fusion de ses filiales régionales ( $c f$. encadré 1).

Source : Enquête ORSEU ( $c f$. encadré 1).

Au-delà de l'incidence psychologique que le salarié subit, ce conflit n'est pas sans conséquence sur la performance commerciale. Un salarié sur deux affirme être incapable de réaliser les objectifs qui lui ont été fixés. Cela peut vouloir dire que ces objectifs sont trop élevés, inadéquats avec ses compétences ou qu'ils sont en opposition avec son éthique. Certains salariés emploient des stratégies de contournement qui consistent à ne pas s'obstiner à vendre des produits qu'ils ne maitrisent pas ou qu'ils estiment peu utiles au client. Ils se contentent de présenter ce type de produits au client sans aller plus loin dans la démarche de vente. D'ailleurs, cette stratégie leur permet de remplir une partie de leurs objectifs en termes de suivi de la clientèle mais elle porte le risque de ne pas remplir les objectifs financiers. Plus de $34 \%$ des commerciaux affirment assumer seuls le poids des décisions et la prise de risque liée à la vente. Cela montre que les stratégies de contournement sont limitées par le partage du risque financier entre le salarié et l'entreprise.

\section{Le management par le benchmarking des performances}

Les objectifs commerciaux en termes de ventes générées sont les mêmes pour toutes les agences. Elles sont déterminées au prorata de leur effectif. De ce fait, les spécificités locales de la clientèle ne sont pas prises en compte. Une agence située dans le milieu rural se voit attribuer les mêmes objectifs qu'une agence de même taille située dans le milieu urbain. Ceci est également le cas entre les agences évoluant dans des milieux sociaux différents (quartier populaire comparé à un quartier à population aisée par exemple). L'uniformisation des objectifs de l'ensemble des agences sans prise en compte des caractéristiques de la clientèle pose le problème de leur réalisabilité pour certaines d'entre-elles. Comme le notent Aude Hubrecht, Michel Dietsch et Fabienne Guerra (2005), le caractère multidimensionnel des activités des agences et la particularité de leur contexte local rendent complexe la comparaison de leur rentabilité. L'harmonisation des objectifs est d'ailleurs perçue par certains salariés comme injuste, ce qui explique qu'un commercial sur deux éprouve des difficultés à réaliser ses objectifs.

$\mathrm{Au}$ niveau de chaque agence, les performances des commerciaux sont suivies au quotidien par le manager à travers l'outil de reporting de l'activité. Cet outil permet au manager de comparer les résultats entre les commerciaux, et au responsable du secteur de comparer les résultats entre les agences sous sa responsabilité. Cette comparaison vise à identifier les agences et les commerciaux modèles d'une part et les agences et les salariés en difficultés d'autre part. Il s'agit d'un management par le benchmarking conduisant à définir une performance modèle comme repère comparatif interne. L'écart de performance par rapport à cette référence est connu par tous les salariés puisque chacun accède à l'information aussi bien concernant les résultats de l'agence que ceux de ses collègues. En effet, les résultats individuels sont exposés quotidiennement au tableau d'affichage à l'intention des salariés. Lors des réunions hebdomadaires entre les équipes de travail, le manager revient sur ces résultats et invite les salariés à commenter leurs performances et leurs difficultés, ce qui peut être une épreuve difficile pour ceux qui sont à la traine. Au sentiment d'infériorité peut s'ajouter la culpabilisation vis-àvis du collectif : comme les primes sont assises sur le taux de réalisation des objectifs de l'agence, les «mauvais commerciaux» impactent négativement ce taux et par conséquence le montant de la prime distribuée.

Ce type de management par le benchmarking peut provoquer chez certains salariés une perte de l'estime de soi synonyme de dévalorisation. Le modèle de régression logistique ( $c f$. tableau 3 ) 
montre que le risque de stress est multiplié par 1,5 chez les salariés confrontés à cette situation. Derrière ce résultat, c'est peut-être toute la démarche d'évaluation de la performance qui est mise en cause. Ce que les salariés critiquent n'est pas le fait qu'on leur demande de réaliser un chiffre d'affaires mais c'est la déconnexion entre les objectifs prescrits et les conditions dans lesquelles on leur demande de les réaliser. L'absence de reconnaissance de ces conditions est l'une des principales causes de la souffrance au travail selon Christian Du TERTRE (2010).

\section{Le soutien social affaibli par la charge de travail, la faible autonomie et les exigences psychologiques}

Plus de $30 \%$ des salariés du réseau affirment «travailler dans une ambiance tendue marquée par des conflits de personnes». Cette proportion est de $47 \%$ chez les salariés stressés. Les deux principaux problèmes à l'origine de la dégradation des relations de travail sont les inégalités de reconnaissance et la mise en concurrence entre les salariés via un management centré sur les objectifs. Le premier problème semble ne pas être lié à la fusion-réorganisation. Les salariés ont souvent évoqué le sujet de promotion fondée sur les affinités de personnes plutôt que sur la motivation et le mérite. Concernant le second problème, les salariés rencontrés n'arrivent pas à donner un véritable sens aux conflits interpersonnels engendrés par le management par les objectifs. Ils affirment que cette nouvelle forme de management contribue plus à la mésentente qu'au bon fonctionnement du collectif de travail. Une certaine opposition est perceptible entre les commerciaux jeunes qui ne remettent pas en cause le management par les objectifs et ceux qui s'y opposent, essentiellement des salariés âgés et plus anciens dans l'entreprise.

Cette ambiance a-t-elle un effet sur le soutien social? Plus de deux tiers des commerciaux interrogés affirment qu'ils peuvent «compter sur le soutien de leurs collègues en cas de difficultés». Cependant, 37,4 \% de ceux qui estiment que l'ambiance au travail est tendue affirment ne pas compter sur le soutien des collègues. Parmi eux, $76 \%$ perçoivent un stress élevé ou très élevé. Pour ces salariés, le soutien social n'est plus donc une ressource mobilisable leur permettant de faire face au stress. La régression logistique montre que le risque de stress est multiplié par $1,8 \mathrm{chez}$ cette catégorie de travailleurs comparativement à ceux pouvant disposer du soutien social des collègues. Le manque de soutien social est de fait le troisième facteur de risque le plus important en termes de contribution au stress dans le réseau. Le débordement de la charge de travail et la faible autonomie viennent en outre limiter la disponibilité des salariés à aider leurs collègues en difficultés. Et la mise en concurrence des conseillers commerciaux dégrade davantage les relations interpersonnelles. Dans le support, le risque associé au soutien social n'est pas significatif. Cela est dû probablement à un collectif de travail plus uni compte tenu de l'effectif moins éparpillé dans le siège que dans les agences.

\section{L'incertitude professionnelle due à l'instabilité de l'organisation et à l'insécurité de l'emploi}

Cette incertitude concerne une très large majorité des salariés des fonctions support. Plus de $78 \%$ «pensent que leur emploi est menacé par les évolutions à venir de leur entreprise». Cette proportion est nettement inférieure chez les commerciaux mais reste à un niveau élevé, soit $63 \%$ d'entre eux. Concernant la perception des salariés de leurs perspectives de carrière, $70 \%$ affirment que «la réorganisation a dégradé leurs opportunités professionnelles ». Sur ce sujet, il n'y a quasiment pas de différences entre les salariés du réseau et ceux du support ${ }^{(5)}$.

Des études empiriques ont démontré que l'insécurité de l'emploi est un facteur important de stress (BurChELl, LAPIDO, WiLKInSON, 2002) dans la mesure où elle renvoie à la dégradation du marché de travail interne. $\mathrm{Du}$ point de vue du modèle déséquilibre effort/récompense (SIEGRIST, 1996), l'insécurité professionnelle est assimilée à l'absence de réciprocité de la part de l'entreprise proportionnellement aux efforts demandés au salarié. Ceci est le cas d'une partie des salariés interrogés, notamment les plus anciens, qui ont fait déjà un effort d'adaptation lors des précédentes restructurations et qui devront faire face aujourd'hui à une dégradation de leurs perspectives d'emploi. Ces efforts en termes de mobilisation et d'engagement au travail étaient justifiés par leur anticipation de la réciprocité en termes de sécurité relative de leur emploi. Dès lors que les gains futurs anticipés compensent les efforts demandés, les salariés faisaient preuve de motivation et de sur-engagement, ce que Johannes SIEGRIST (op. cit.) qualifie de stratégies d'ajustement aux exigences de travail (MATSCHINGER et al., 1986). Or, l'incertitude professionnelle induite par la fusion actuelle limite ces stratégies d'ajustement. Est-il rationnel de poursuivre le sur-engagement quand on anticipe une perte d'emploi ou une mobilité professionnelle contrainte? Les salariés qui sont face à ce type de choix sont en grande majorité des salariés démotivés et stressés. Comme le notent Magnus Sverke, Joanna HellgRen et Katharina NAswall (2006), la perception qu'a un salarié de son avenir professionnel peut

(5) Rappelons que l'enquête est effectuée dans un contexte de crise économique. A la question de savoir si la conjoncture a contribué à la dégradation des conditions de leur emploi, la majorité des salariés ont répondu par l'affirmatif. Il convient donc de ne pas limiter la mauvaise perception de l'avenir professionnel à la seule fusion-réorganisation. 
avoir des conséquences psychosociales aussi négatives que la perte de l'emploi lui-même.

Selon notre enquête, ce facteur d'incertitude professionnelle contribue significativement au stress. Dans le support, la probabilité d'être stressé est multipliée par 3,3 chez les salariés incertains de leur avenir professionnel. Elle est le troisième facteur le plus générateur de stress après la charge de travail et l'impact de la nouvelle répartition du travail. Le réseau n'est pas épargné par cette incertitude bien que la fusion se soit accompagnée de la création de nouveaux postes. Mais les commerciaux anticipent une nouvelle réorganisation dans le réseau qui aurait un impact sur les emplois, et ce, même si ce projet n'a jamais été confirmé par la direction. Cette perception négative de leur avenir professionnel multiplie le risque de stress par 1,7 (toutes choses égales par ailleurs).

\section{L'interdépendance des facteurs de risques psychosociaux : une proposition de lecture dynamique}

Nous l'avons vu dans les analyses de régression, la plupart des facteurs de risque joue indépendamment les uns des autres sur le stress perçu. Toutefois, tout au long de l'analyse, nous avons évoqué l'interdépendance des facteurs de RPS. Nous représentons ici les principales corrélations entre ces facteurs (cf. graphique 7) et en proposons une lecture dynamique à partir de nos observations de terrain.

La fusion-réorganisation poursuit l'objectif d'efficacité organisationnelle et d'optimisation de la stratégie commerciale. La recherche de l'efficacité organisationnelle se traduit par une redistribution des tâches et des responsabilités suite à la suppression de postes, aux mobilités professionnelles et à la migration de certains services vers des plateformes régionales ou nationales. En même temps, les procédures internes et les process ont été modifiés dans le but d'accompagner la nouvelle organisation du travail. Concernant la stratégie commerciale, son optimisation s'est faite par la diversification des produits et de la clientèle accompagnée par la spécialisation des salariés et l'adaptation du management et des procédures de travail.

Les trois évolutions énumérées précédemment modifient significativement les situations de travail et exigent une adaptation individuelle et collective des salariés. Il s'agit d'exigences psychologiques dans le sens où l'effort demandé aux salariés est d'intégrer un nouveau mode de fonctionnement et une nouvelle approche de leur métier. Pour certains salariés, cette exigence est à gérer dans un contexte d'incertitude professionnelle véhiculée par la nouvelle répartition des tâches et des fonctions.

Graphique 7 : L'interaction entre les facteurs de risques psychosociaux liés à la fusion-réorganisation

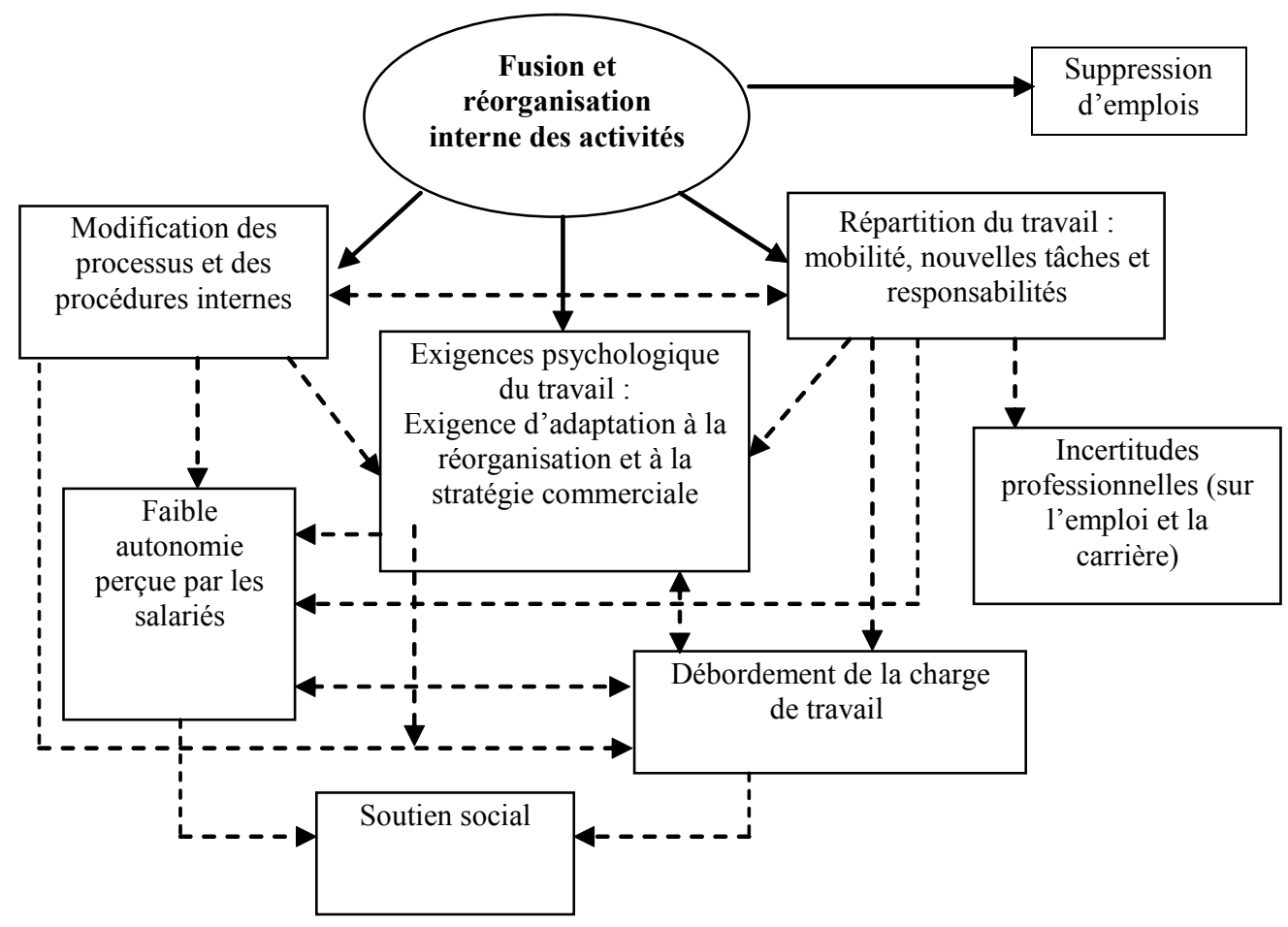

Note : Les corrélations entre les facteurs de RPS sont comprises entre 0,23 et 0,6 . Le seuil critique de significativité pour un échantillon de 1000 individus est de 0,062 avec un intervalle de confiance de $95 \%$ (table de Bravais-Pearson). Ce schéma ne présente que les corrélations dont le coefficient $r>0,3$.

Champ : Salariés d'un groupe financier ayant opéré une fusion de ses filiales régionales ( $c f$. encadré 1).

Source : Enquête ORSEU ( $c f$. encadré 1). 
Outre l'exigence d'adaptation, ces évolutions impliquent un surcroît de la charge de travail perçue. Celle-ci se matérialise par une pression par les objectifs chez les commerciaux tandis que dans le support, la charge de travail est due à la réduction de l'effectif, à l'absentéisme et à la pression provenant des commerciaux. Les salariés doivent ainsi gérer à la fois l'exigence de leur charge de travail et l'exigence d'adaptation que demande l'évolution de l'organisation interne.

La conjugaison de ces facteurs limite les aptitudes à faire face aux nouvelles exigences et notamment l'autonomie, ce qui est synonyme d'un déséquilibre entre les ressources disponibles et les contraintes perçues. Du point de vue de l'approche transactionnelle, l'autonomie réelle dans le travail pourrait atténuer les effets de ce déséquilibre. Or, une grande partie des salariés affirme avoir perdu en autonomie à cause de leur confrontation à un nouvel environnement de travail qu'ils ne maîtrisent pas ou peu. D'ailleurs, le risque associé à la perte d'autonomie est fortement corrélé avec les autres facteurs de RPS. Ce résultat rejoint l'interprétation de l'approche transactionnelle selon laquelle le stress est accentué par la faible marge de manœuvre du salarié dans son travail. Cette même marge de manœuvre est restreinte par l'affaiblissement du soutien social : la disponibilité des salariés à soutenir leurs collègues en difficulté décline avec la dégradation du contexte psychosocial du travail conjuguée à une surcharge de travail et à une faible autonomie.

L'interdépendance des facteurs de RPS présentée ici n'est pas synonyme d'une causalité systématique, mais plutôt d'un enchainement de conséquences provoqué par la «coévolution» de plusieurs aspects de l'organisation. En effet, les situations de travail subissent une modification par la convergence de différentes évolutions internes qui s'influencent mutuellement. L'interdépendance des facteurs de RPS limite l'efficacité des stratégies de coping, ce qui aurait amplifié le stress ou prolongé sa durée.

\section{Limites et perspectives}

L'analyse croisée entre la PSS et les facteurs de RPS a permis de lier le stress perçu aux exigences de travail induites par la réorganisation. L'enquête a montré que les salariés exposés à ces facteurs sont les plus stressés. Une première limite de ce travail doit cependant être mentionnée, qui tient aux données mobilisées : l'échelle de stress perçu et les facteurs de risques psychosociaux étant mesurés au travers des déclarations des salariés effectuées dans une seule et même enquête, on peut craindre que leur objectivation ne soit que partielle et que la liaison entre les deux soit endogène. Cette limite ne résiste toutefois pas à l'analyse puisque les observations et entretiens conduits dans l'entreprise viennent très largement confirmer les déclarations effectuées dans l'enquête. Une autre limite peut être formulée, qui fournit en même temps des perspectives d'analyse complémentaires à cette recherche : une partie de l'échantillon qui a les mêmes caractéristiques socioprofessionnelles que les salariés stressés (ancienneté, âge et qualification) ne présente pas de stress bien qu'elle soit exposée aux mêmes facteurs de RPS. L'enquête ne permet pas de savoir si cette différence est due aux caractéristiques idiosyncrasiques de ces salariés ou à des ressources organisationnelles spécifiques qu'ils ont pu mobiliser pour conduire des stratégies de coping efficaces. Un complément d'informations sur les réponses adoptées par ces salariés pourrait améliorer la compréhension de la problématique du stress au travail.

$$
\text { * } \quad \text { * }
$$

L'expérience de la fusion analysée dans cet article illustre comment le changement organisationnel qui devait améliorer l'efficience globale de l'organisation devient un creuset de risques psychosociaux.Au terme de l'enquête, les partenaires sociaux ont négocié un plan d'actions pour lutter contre ces risques. Les principales actions visent à réduire la portée des facteurs de RPS identifiés. Cependant, l'efficacité de ces actions sera réduite si l'organisation ne s'engage pas dans une stabilité durable. La succession de projets de réorganisations crée en effet une exigence d'adaptation permanente des salariés. Le manque de visibilité sur l'avenir suite à ces changements fragilise leurs rapports de confiance avec leur entreprise et crée ainsi un climat d'insécurité professionnelle, notamment pour les moins qualifiés et les plus âgés. Les résultats de l'enquête sont édifiants : $66 \%$ des salariés sont incertains quant à leur avenir professionnel.

Sans doute la conjoncture de crise économique a eu un impact sur les réponses des salariés. Mais nous pensons que le risque de certains facteurs aurait pu être limité si une projection économique et professionnelle claire leur avait été proposée. L'existence d'une telle projection aurait même permis d'anticiper les changements et de mettre en place un programme d'adaptation des compétences aux métiers qui se développent en interne. Un marché interne dynamique aurait alors fourni une perspective pour les stratégies d'adaptation des salariés. Or, aucun accord sur la gestion prévisionnelle des emplois et des carrières n'a été signé, ni même négocié par les filiales fusionnées. Cela révèle, et c'est le sentiment des salariés rencontrés, l'absence de prise de conscience au plus haut niveau de la direction du groupe sur les risques psychosociaux que les fusions-réorganisations engendrent. 


\section{Bibliographie}

Bellinghausen L., Collange J., Botella M., Emery J-L., Albert E. (2009), «Validation factorielle de l'échelle française de stress perçu en milieu professionnel», Santé publique, $\mathrm{n}^{\circ} 21$.

Bellotto M., Colautti C. (1987), “The psychosocial diagnostic of organizational culture", Feelings in Europe, vol. 5 .

Boltanski L., Chiapello E. (1999), Le nouvel esprit du capitalisme, Paris, Gallimard.

Burchell B., Lapido D., Wilkinson F. (2002), Job Insecurity and Work Intensification, Routledge, London.

Cohen S., Kamarck T., Mermelstein R. (1983), “A Global measure of perceived stress", Journal of Health and Social Behavior, $\mathrm{n}^{\circ} 24$.

Cohen S., Williamson G. (1988), "Perceived stress in a probability sample of the United States", in Spacapan S, Oskamp S, (dir.), The social psychology of health : Claremont Symposium on applied social psychology. Newbury Park, CA, Sage.

COMITÉ DES ÉTABLISSEMENTS DE CRÉDIT ET DES ENTREPRISES D'INVESTISSEMENT (2009), Rapport annuel, CECEI.

DARES (2009), Indicateurs provisoires de facteurs de risques psychosociaux au travail, Rapport au ministre du Travail, de la Solidarité et de la Fonction publique.

Du Tertre C. (2010), «De l'évaluation individuelle à la souffrance», Santé et Travail, $\mathrm{n}^{\circ} 71$.

Gadea C., Cercle A. (2004), «Le stress perçu et son évaluation en milieu professionnel : intérêts et limites de l'échelle PSS», in Lancry A, Lemoine C. (Eds), La personne et ses rapports au travail, Paris, L'Harmattan.

Gervais M., Thenet G. (2004), «Comment évaluer la productivité dans les activités de Service?», Comptabilité Contrôle, Audit, tome 10, vol. 1.

HIRES (2010), Santé et restructurations, DG Emploi et affaires sociales de la Commission européenne.

Hubrecht A., Dietsch M., Guerra F. (2005), «Mesure de la performance des agences bancaires par une approche DEA», Finance-Contrôle-Stratégie, vol. 8, nº 2.

InSERM (2011), Stress au travail et santé. Situation chez les indépendants, Les éditions Inserm, Paris.

Jex S., BeEHR T.A. (1991), "Emerging theoretical and methodological issues in the study of workrelated stress", in Rowland K, Ferris G, (Eds.), Research in Personnel and Human Resources Management, CT, JAI Press, Greenwich.

Koleck M., Quintard B., TAstet S. (2002), "French validation of the Perceived Stress Scale: the construct validity", $16^{e}$ Conférence de l'European Health Psychology Society, Lisbonne, Portugal.

Lazarus R.S., Folkman S. (1984), Stress, appraisal and coping, New York, Springer.
LEIBENSTEIN H. (1972), "On the basic proposition of X-Efficiency Theory", American Economic Association, Efficiency of Managerial Decision Process, May, vol. $n^{\circ}$ 68-2.

MAtschinger H. et al. (1986), "Type A as a Coping Career: Toward a Conceptual and Methodological Redefinition", in Schmidt T.H., Dembrowski T.M., et Blümchen G., (Eds.), Biological and Psychological Factors in Cardiovascular Disease, Springer, Berlin.

Neboit M., Vèzina M. (dir.) (2007), Stress au travail et santé physique, Octarès Éditions, Toulouse.

Neufeld R.W.J., Paterson R.J. (1989), "Issues concerning control and its implementation", in Neufeld RW. J, (dir.), Advances in the investigation of psychological stress, Wiley, New York.

Newton T.J. (1989), “Occupational stress and coping with stress: a critique”, Human Relations, vol. 42, n 5.

Organisation IntERnationale DU Travail (2001), L'incidence sur l'emploi des fusions et des acquisitions dans le secteur des services bancaires et financiers, OIT, Genève.

ORgANISATION MONDIALE DE LA SANTÉ (2004), «Organisation du travail et stress», Rapport, Série Protection de la santé des travailleurs, $\mathrm{n}^{\circ} 3$, OMS, Genève.

Ory J.N., GuRTnER E., JAEGER M. (2006), «Les enjeux des mutations récentes des groupes bancaires coopératifs en France», RECMA, $\mathrm{n}^{\circ} 301$.

PonNelle S., Lancry A. (2007), «Stratégies d'ajustement et ressources environnementales et personnelles dans la dynamique du stress », in Neboit M., Vèzina M. (dir.), Stress au travail et santé physique, Octarès Éditions, Toulouse.

PASTRÉ O. (1993), «Le système bancaire français, bilan et perspectives », Revue d'Économie Financière, n 27.

Richez-Battesti N., Gianfaldoni P. (dir.) (2005), Les banques coopératives en France : entre banalisation et renouveau des spécificités? Rapport, Délégation interministérielle à l'Économie sociale.

Siegrist J. (1996), “Adverse Health Effects of High-Effort/ Low-Reward Conditions", Journal of Occupational Health Psychology, vol. 1, n 1 .

Sverke M., Hellgren J., Naswall K. (2006), Job Insecurity : A literature review, National Institute for Working Life, Stockholm.

Suls J., Fletcher B. (1985), "The relative efficacy of avoidant and non-avoidant coping strategies: A metaanalysis", Health Psychology, 4, pp. 249-288.

Thenet G. Guillouzo R. (2003), «Benchmark et mesure de performance du secteur bancaire : Une application de la méthode DEA», La Revue des Sciences de Gestion, n 203.

VÈZINA M. (2007), «Stress au travail et santé psychique : rappel des différentes approches», in Neboit M., Vèzina M. (dir.), Stress au travail et santé physique, Octarès Éditions, Toulouse. 


\section{Annexe}

Les réponses aux questions constitutives de l'échelle de la PSS (en \%)

\begin{tabular}{|c|c|c|c|c|c|}
\hline Au cours du dernier mois, à quelle fréquence : & Jamais & $\begin{array}{l}\text { Presque } \\
\text { jamais }\end{array}$ & Parfois & $\begin{array}{c}\text { Assez } \\
\text { souvent }\end{array}$ & $\begin{array}{c}\text { Très } \\
\text { souvent }\end{array}$ \\
\hline 1 - avez-vous été perturbé(e) par des événements inattendus? & 8 & 18 & 39 & 26 & 10 \\
\hline $\begin{array}{l}2 \text { - avez-vous eu l'impression de ne pas pouvoir contrôler ce qui est } \\
\text { important dans votre vie? }\end{array}$ & 14 & 23 & 33 & 22 & 8 \\
\hline 3 - vous êtes-vous senti(e) nerveux (se) ou stressé(e)? & 3 & 8 & 31 & 33 & 25 \\
\hline $\begin{array}{l}4 \text { - vous êtes-vous senti(e) en mesure d'aborder vos problèmes } \\
\text { personnels avec sérénité? }\end{array}$ & 3 & 16 & 35 & 35 & 11 \\
\hline $\begin{array}{l}5 \text { - avez-vous senti que les événements se déroulaient comme vous le } \\
\text { souhaitiez? }\end{array}$ & 3 & 21 & 39 & 33 & 5 \\
\hline $\begin{array}{l}6 \text { - vous êtes-vous senti(e) dépassé (e) par tout ce que vous aviez à } \\
\text { faire? }\end{array}$ & 6 & 18 & 36 & 27 & 12 \\
\hline $\begin{array}{l}7 \text { - vous êtes-vous senti(e) capable de maîtriser les désagréments que } \\
\text { vous rencontriez? }\end{array}$ & 2 & 11 & 39 & 40 & 8 \\
\hline 8 - vous êtes-vous senti(e) au mieux de vos capacités? & 2 & 13 & 39 & 40 & 7 \\
\hline $\begin{array}{l}9 \text { - vous êtes-vous senti(e) énervé(e) parce que les événements } \\
\text { échappaient à votre contrôle? }\end{array}$ & 5 & 17 & 37 & 30 & 12 \\
\hline $\begin{array}{l}10 \text { - avez-vous senti que les difficultés étaient tellement énormes que } \\
\text { vous ne parvenez pas à les maîtriser? }\end{array}$ & 25 & 29 & 28 & 12 & 6 \\
\hline
\end{tabular}

Note : Les questions sont traduites à partir de CoHEN, Williamson (1988). Pour la construction de la PSS, les modalités «jamais» à «très souvent» sont cotées de 0 à 4 pour les questions 1,2,3,6,9 et 10. Les scores sont inversés pour les questions $4,5,7$ et 8 . La valeur de la PSS est obtenue par l'addition des scores des 10 questions.

Lecture : $8 \%$ des répondants affirment qu'ils n'ont jamais été perturbés par un événement inattendu au cours du dernier mois précédent l'enquête.

Champ : Salariés d'un groupe financier ayant opéré une fusion de ses filiales régionales ( $c f$. encadré 1).

Source : Enquête ORSEU ( $c f$. encadré 1). 